\title{
ESTUDO DAS TEMPERATURAS POR TERMOGRA- FIA EM AMOSTRAS SUBMETIDAS A ENERGIAS DE RAIOS X
}

\author{
Gladis Aparecida Galindo Reisemberger de Souza', Ramón Sigifredo Cortés Paredes², Frieda Saicla \\ Barros $^{3}$, Carlos Dalmaso Neto ${ }^{4}$, Marcos Leal Brioschi ${ }^{5}$
}

1 Doutoranda, PGMEC, Universidade Federal do Paraná (UFPR)

2 Professor Doutor, PGMEC Universidade Federal do Paraná (UFPR)

3 Professora Doutora, Universidade Tecnológica Federal do Paraná (UTFPR)

${ }_{4}^{4}$ Médico. Mestrando, Pós-graduado em Termologia e Termografia, (FMUSP)

${ }^{5}$ Médico Professor, Doutor. Professor da Pós-graduação em Termologia e Termografia, (FMUSP)

Resumo: Este trabalho apresenta comparações de energia radiante (quantidade de energia emitida por um objeto por unidade de tempo (W)) pela tecnologia de infravermelho, denominada Termografia, antes e depois de testes de raiox $\mathrm{X}$ em amostras preparadas para blindar radiação $\mathrm{X}$ em clinicas, hospitais e locais onde se utilizam radiações ionizantes para realização de exames médicos nos pacientes em investigação de saúde. 0 objetivo deste estudo é a verificação da movimentação molecular para observação de atenuação em placas revestidas com pó de chumbo e pó de barita pela tecnologia de Aspersão Térmica. Como metodologia, foi utilizada uma câmera de Termografia que captou imagens dentro de um equipamento de raios $x$ utilizado para o arranjo experimental do estudo, as medidas foram realizadas em algumas amostras com aplicações de vários valores de energias, kilovoltagem (kV), antes e após os raios $X$ serem emitidos nas peças. Os resultados dessas medições demonstraram que o método de dissipação primário nas peças é por convecção externa, e que a energia emitida pelas amostras sofre mudanças, para comparação com atenuação de raios $X$..

Palavras-chave: termografia; aspersão térmica; raios $x$; atenuação.

Abstract: This paper presents radiant energy comparisons (amount of energy emitted by an object per unit of time $(W)$ ) by infrared technology called Thermography, before and after $X$-ray tests on samples prepared to shield $\mathrm{X}$-rays in clinics, hospitals and places where ionizing radiation is used to perform medical examinations on patients in health investigation. The objective of this study is to verify the modification of temperatures at times of application of $X$-rays on materials, being thermographic images performed during attenuation tests on tiles that have the purpose of shielding X-radiation. As a methodology, a thermography camera was used. captured images inside x-ray equipment used for the experimental arrangement of the study, the measurements were made in some samples with applications of various values of energy, kilovoltage $(\mathrm{kV})$, before and after the $\mathrm{x}$-rays were launched in the pieces. The results of these measurements demonstrated that the primary dissipation method in the parts is by external convection, and that the energy emitted by the samples changes, compared to $X$-ray attenuation.

Keywords: thermography; thermal spray; $X$ ray; attenuation 


\section{PAN AMERICAN JOURNAL OF MEDICAL THERMOLOGY}

\section{INTRODUÇÃO}

Em clinicas, hospitais e ambientes onde são utilizadas as radiações ionizantes, métodos de blindagem são fundamentais para a proteção de pessoas que circulam em ambientes próximos, que trabalham ou se expõe de qualquer outra forma nesses locais ${ }^{1}$.

Segundo o pesquisador John B. Rulssel ${ }^{2}$, atualmente são utilizadas placas de chumbo no meio da construção das paredes de clinicas e hospitais, ou argamassa baritada, entretanto, quando há necessidade de demolição das paredes nesses locais, o assunto torna-se bastante complexo, o que impossibilita o serviço no local, por um período longo.

$\mathrm{Na}$ expectativa da inovação, pesquisadores estão em constante busca de maneiras e práticas funcionais com resultados de qualidade, que objetivem melhorias nos materiais utilizados em várias áreas. É necessário a compreensão de novos materiais, cuja pesquisa volta-se para as áreas de saúde, proteção radiológica e engenharias.

Este estudo fez parte de uma análise de proteção radiológica envolvendo chumbo e barita em pó para revestimento em azulejo de cerâmica crua com intuito de atenuar radiação ionizante nos ambientes já mencionados anteriormente. Um dos objetivos foi a obtenção de facilidade na manutenção ou nova obra relacionada com blindagem de paredes em locais utilizados na área de saúde, para exames com radiação ionizante.

As amostras do material do estudo foram produzidas o com a utilização do método de Aspersão Térmica (AT), o qual reveste materiais com a utilização de pós ou arames. Aspersão Térmica (AT) é uma tecnologia com uma variedade de processos em que materiais metálicos ou não-metálicos, no formato de pó, vareta, cordão ou arame, são depositados em uma característica fundida ou semifundida sobre um substrato, este é preparado para fundamento de um depósito aspergi$\mathrm{do}^{3}$. Casteletti et al. ${ }^{4}$, afirma que esta tecnologia se compõe de vários processos, técnica versátil que realiza deposições em superfícies diversas para obtenção de materiais revestidos.

O objetivo maior desta pesquisa é a verificação das energias radiantes (W) emitidas do material (amostras) de blindagem de radiação ionizante, a fim de comparar com a atenuação verificada nas mesmas amostras, quando nestas, foram enviadas energias $(\mathrm{kV})$, utilizando a tecnologia de imagens termográficas, com câmera apropriada. A Termografia é uma técnica que capta a radiação infravermelha emitida pelos corpos, possibilitando a medição de temperaturas e a observação de padrões de distribuição de calor em um determinado sistema. Existe uma vasta gama de aplicações da termografia em diversas áreas, tais como: militar, medicina, indústria e engenharia ${ }^{5,6}$.

Esta emissão de radiação depende da emissividade dos materiais e varia com o comprimento de onda e com a temperatura ${ }^{7}$. A Lei de Stefan-Boltzmann define a relação entre a energia irradiada e temperatura ao afirmar que o total de radiação emitida por um objeto é diretamente proporcional à sua área. A emissividade, $\varepsilon$ da pele humana é bastante alta $(\varepsilon$ pele $=0,98$, sendo que um corpo negro tem $\varepsilon$ corpo negro $=1)^{8}$.

Atenuação de um material, refere-se a um feixe de raio $x$ ou gama quando interage com um material de espessura $x$, parte do feixe é espalhada, parte dele é absorvida e uma pequena fração atravessa o material sem que haja interação,quanto mais espesso é o material, menor será a energia da radiação que passa pelo material após a travessia. Quanto maior a energia 
dos fótons da radiação incidente, maior será também a sua capacidade de penetração $0^{9,10}$.

Relacionado com o trabalho realizado por Novo, M.M.M. et al.e Holman ${ }^{11}$, a transferência de calor, extremamente importante para a obtenção de valores e ocorre por meio de três mecanismos: condução, convecção e radiação ${ }^{12,13}$.

\section{Metodologia}

Os termogramas com as imagens utilizadas para o ensaio, foram feitos através de uma câmera termográfica Therma CAM T400 (FLIR Systems Inc, North Billerica, Suécia), que possui um detector do tipo uncooled focal plane (FPA), i.e., micro bolômetro sem refrigeração de quarta geração com 320x240 pixels (76.800 pixels), que atua na faixa espectral de ondas eletromagnéticas entre 7,5 a $13 \mu \mathrm{m}$, o que corresponde à faixa do infravermelho longo (FIR) para estudo na faixa de temperatura $-20^{\circ} \mathrm{C}$ até $+350^{\circ} \mathrm{C}$. O equipamento permite obter imagens com resolução espacial (IFOV) de 1,4 mrad, para visualização de pontos quentes de $1,4 \mathrm{~mm}$ a distâncias de 1 metro, com lente padrão e sem lentes adicionais. A precisão de temperatura reportada pelo fabricante é de $\pm 1^{\circ} \mathrm{C}$ e resolução de temperatura de $0,05^{\circ} \mathrm{C}$ a $30^{\circ} \mathrm{C}$.

O programa software utilizado para encontrar as medidas máximas de energia de potencia, foi o Flir Tools, capaz de modificar a emissividade, dependendo

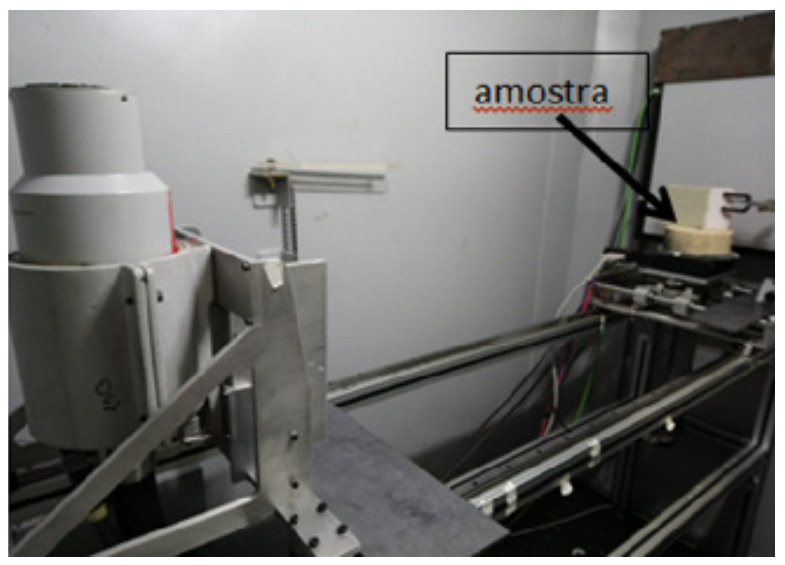

Figura 1 - Equipamento de raios $X$ do material utilizado e para este trabalho foi aplicada a medida de energia radiante, que é a quantidade de energia emitida por um objeto por unidade de tempo (W), neste estudo houve uma mistura de materiais e não se poderia quantificar um valor para emissividade, no software, então foi utilizado a energia radiante (W), segundo as equações de Stefan-Boltzmann, já mencionadas anteriormente neste artigo. A equação de Boltzmann, na câmera de termografia, pode transformar a energia radiante para temperaturas em graus CeIcius, quando se menciona a emissividade do corpo a ser estudado.

Durante as aplicações de energias (kV) nas amostras, para verificação de atenuação do material, foram feitas imagens termográficas para a análise das diferenças de calor nos materiais, após a produção dos raios $\mathrm{X}$ enviados nas amostras. Na Figura 1, a imagem do equipamento de raios X utilizado para medir atenuação dos matérias com a amostra.

\section{Resultados e Discussão}

Nos resultados do estudo Termográfico para verificação das diferentes energias radiantes (W) pode-se verificar que as temperaturas das amostras estudadas tiveram modificações das suas energias. Os resultados dessas medições demonstraram que o método de dissipação primário nas peças é por convecção externa e que a o valor da intensidade de potência tem um sensível aumento, conforme a atenuação é mais eficiente. A Figura 2 ilustra a peça antes de ser irradiada com raios $X$. A Figura 3 mostra a variação da intensidade depois da irradiação.

A primeira imagem analisada, chamada de Figura $\mathbf{3 A 1} \mathrm{com}$ aplicação de 100 $\mathrm{kVs}$ de energia, amostra com a espessura de $90 \mathrm{~mm}$., demonstra $142.6 \mathrm{~W}$ de energia radiante, a figura B1 da esquerda, com emissão de 180 kVs e com a mesma es- 


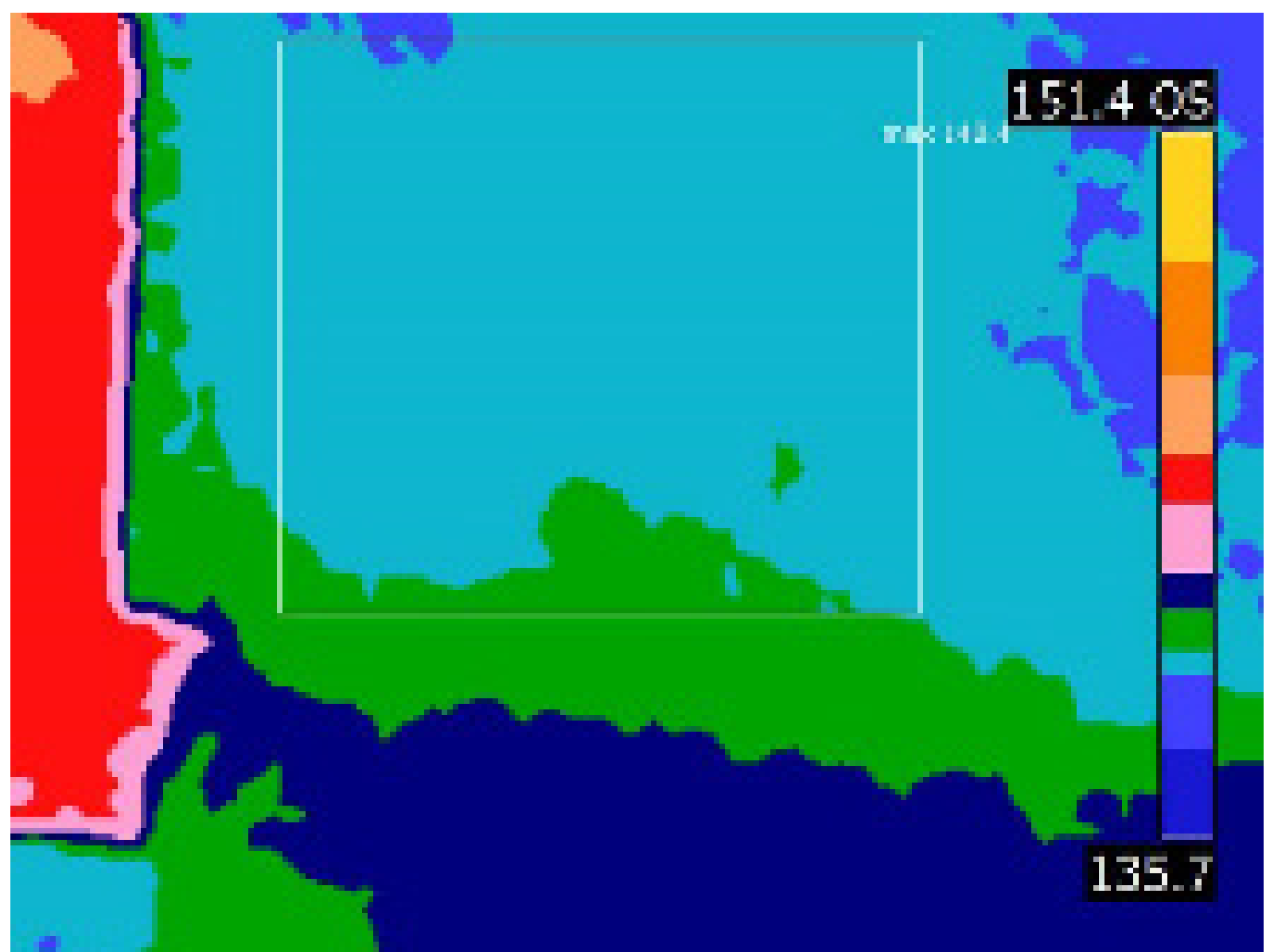

Figura 2 - Termograma com análise de energia radiante da amostra antes de ser submetida as energias de Raios $\mathrm{x}$

pessura, mostra 141.4 W de intensidade de potência, a Figura 3A2 com espessura de $8,1 \mathrm{~mm}$. que sofreu emanação de 100 kVs possui 142.1W de potência energética e a Figura 3B2 com energia emitida de $100 \mathrm{kVs}$ e espessura $8,1 \mathrm{~mm}$. ilustra uma energia radiante de 141.1W.

Pode-se perceber que quanto maior a espessura do material, mais atenuação ocorre e demonstra que as moléculas estão em maior movimentação e que quanto menor é a energia de raios $\mathrm{X}(\mathrm{kV})$ emanada na amostra, maior também é a atenuação, demonstrando que a movimentação das moléculas é significativamente maior. Isto significa que quando a atenuação é eficiente, a energia (raios X) fica armazenada na amostra, já que grande parte da energia $X$ não atravessou o material.

\section{CONCLUSÃo}

Neste estudo, conclui-se que existe mudança significativa de potência energética em amostras que foram submetidas a energias diferentes de raios $X(k v)$. Sendo as energias de dissipação pri-mária nas peças, por convecção externa. Atenden-do aos objetivos deste estudo conclui-se que as moléculas sofrem movimentação maior quando a atenuação é mais eficiente, em placas revestidas com pó de chumbo e pó de barita pela tecnologia de Aspersão Térmica. .

\section{REFERÊNCIAS}

1. Reisemberger, G.G. Proposta de tratamento com misturas de pó de chumbo e pó de barita em materiais cerâmicos utilizados para blindagem de radiação ionizante por meio do processo de aspersão térmica. Tese para titulo de doutor em Engenharia Mecânica. UFPR. Fevereiro, 2020. (Ainda não publicado).

2. RUSSELL, John B.; Química Geral vol.1, São Paulo: Pearson Education do Brasil, Makron Books,1994. 


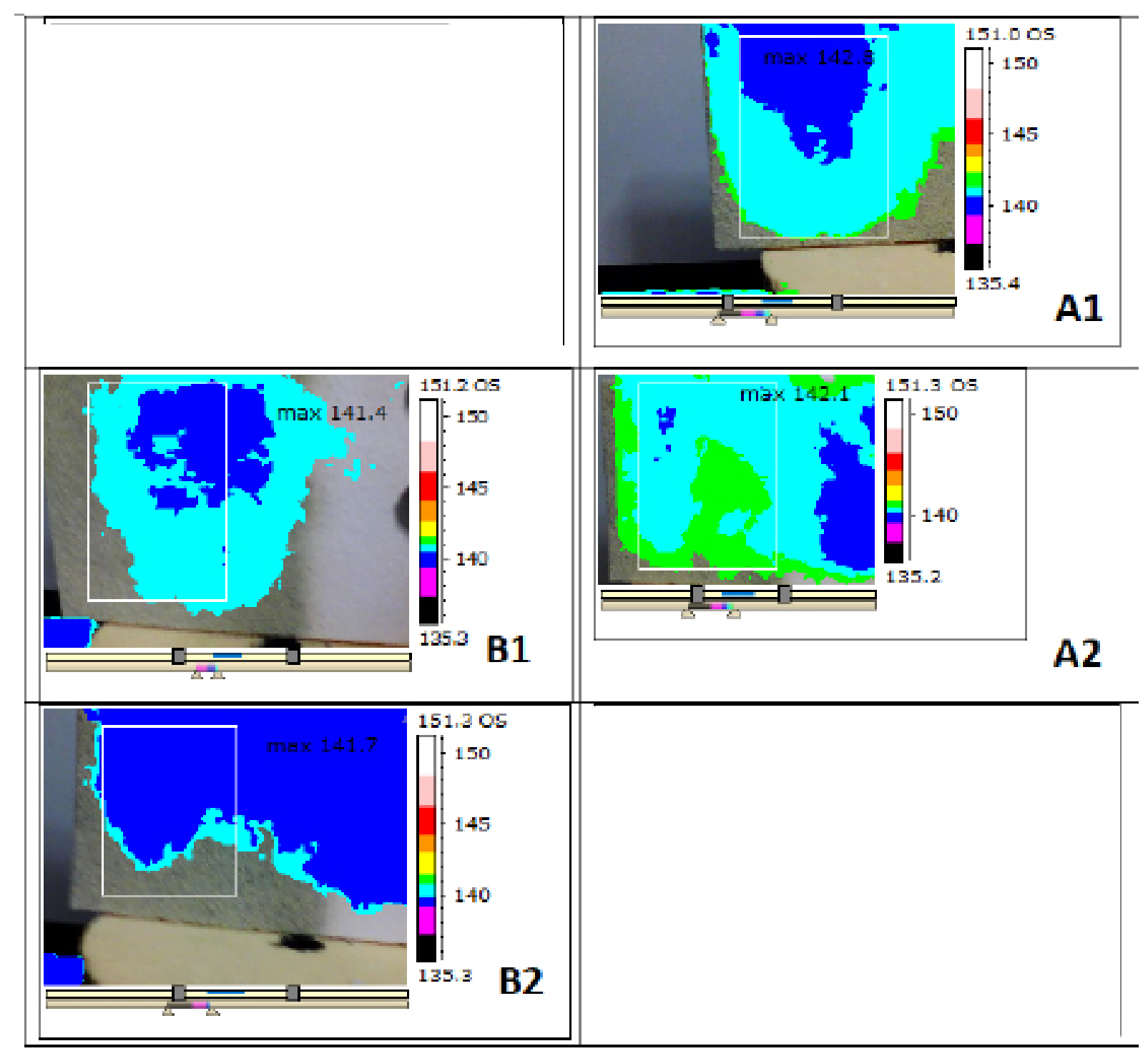

Figura 2 - Imagens com medidas de intensidade de potência

3. Paredes, R. S. C; D'Oliveira, A. S. C. M.; Amico, S. The Effect of Rugosite and substratum Pre-heating on the Morphology of Aluminum Coating Deposited by Thermal Spray.Surface and Coating Technology, Elsevier, v. 200, p. $3050-3055,2006$

4. Casteletti, L. C., Fernandes, F. A. P., Takeya, G. S., Picon, C. A.,Tremiliosi-Filho, G. Avaliação da resistência à corrosão do aço AISI 420 depositado por processos variados de aspersão térmica. Rem: Revista Escola de Minas, v. 63, p. 87-90, 2010

5. Altoé, L, Oliveira Filho, D. Termografia infravermelha aplicada à inspeção de edifícios. Acta Tecnológica; 7: 5559,2012

6. Reisemberger, G.G. Analise e triagem de tumor benigno a partir de imagens de infravermelho. Dissertação (Mestrado em Engenharia Mecânica) - Setor de Tecnologia, Universidade Federal do Paraná, Curitiba, 2014.

7. Gratt BM, Sickles EA. Electronic facial thermography: an analysis of asymptomatic adult subjects. J Orofac
Pain. 1995; 9(5): 255-265

8. BEJAN, A. Heat Transfer, $1^{\text {a }}$ edição. New York: Wiley, 1993

9. EVANS R.D. The atomic Nucleus. USA; Robert E. Krieger Publishing Company, Inc; 1982.

10. TAUHATA, L.; SALATI, I.; DI PRINZIO, R.; DI PRINZIO, A. - Radioproteção e Dosimetria: Fundamentos - 10a revisão - IRD/CNEN. 344p. Rio de Janeiro. Abril /2014.

11. M. M. M. Novo, C. S. Bitencourt, P. R. T. Tiba, D. G. M. Silva, V. C. Pandolfelli. Fundamentals on emissivity and its correlation with the refractory materials, energy saving and the environment, Cerâmica 60 (2014) 22-33.

12. J. P. Holman, "Transferência de calor", McGraw-Hill do Brasil, S. Paulo, SP (1983) 639p.

13. F. P. Incropera, D. P. DeWitt, "Transferência de Calor e de Massa", $5^{a}$ Ed., LTC, Rio de Janeiro, RJ (2002) 698p. 